\title{
Gangrenous Amyand's hernia in neonate: A great clinical masquerader
}

\author{
Vaibhav Pandey*, Ajar Narayan Gangopadhyay, Dinesh Kumar Gupta, Shiv Prasad Sharma
}

Department of Paediatric Surgery, Institute of Medical Sciences, Banaras Hindu University, Varanasi, India

Email: ${ }^{*}$ sunny.imsbhu@gmail.com

Received 14 August 2013; revised 12 September 2013; accepted 20 September 2013

Copyright (c) 2013 Vaibhav Pandey et al. This is an open access article distributed under the Creative Commons Attribution License, which permits unrestricted use, distribution, and reproduction in any medium, provided the original work is properly cited.

\begin{abstract}
Amyand's hernia $[\mathrm{AH}]$ is a rare condition with presence of vermiform appendix in an inguinal hernia sac [1]. It is a rare entity with very difficult preoperative diagnosis that does not affect management. We herein report a neonatal gangrenous $\mathrm{AH}$ masking all features of underlying bowel gangrene. In neonates, a great masquerader can mask all the features of underlying bowel gangrene and delay in diagnosis can be detrimental in context of outcome. A high index of suspicion of Amyand's hernia with gangrene should be kept even if child presents with irreducible hernia without features of strangulation to prevent avoidable increase in morbidity and mortality.
\end{abstract}

Keywords: Hernia; Gangrenous Appendix;

Strangulation; Inguinal; Appendicitis

\section{INTRODUCTION}

Amyand's hernia is a rare condition with presence of vermiform appendix in an inguinal hernia sac [1]. Preoperative diagnosis of Amyand's hernia is very difficult and it is often misdiagnosed as incarcerated [1]. We herein present a case of neonatal gangrenous Amyand's hernia masquerading clinical features of strangulation.

\section{CASE REPORT}

A 25-day male baby, who had a right-sided reducible inguinal hernia since birth, presented with a 2-dayhistory of sudden increase in size and irreducibility of the hernia. There was no history of vomiting or abdominal distension and child was taking feeds normally. On examination child was active, afebrile with heart rate of 134 beats per minute. Abdomen was soft with normal bowel sounds. There was a pear-shaped right inguinoscrotal swelling which was tense, tender, and irreducible with normal overlying skin (Figure 1). Diagnosis of right irreducible inguinal hernia was made. X-ray abdomen was normal. Child was started on intravenous antibiotics, fluids and prepared for surgery. Child was explored after 24 hours of admission, on opening the hernial sac through right inguinal skin crease incision, gangrenous appendix was found to be lying within, with dense adhesions to the sac. The incision was extended towards scrotum and appendix of size $4 \mathrm{~cm}$ was released (Figure 2). Appendicectomy and herniotomy were performed and the patient transferred to the ward, where he had an uneventful postoperative stay.

\section{DISCUSSION}

The presence of an appendix within the inguinal hernial sac is defined as Amyand's hernia. The incidence of having a normal appendix within an inguinal hernial sac is about $1 \%$, whereas only $0.1 \%$ of all cases of appendicitis present in an inguinal hernia [2]. Contrary to appen-

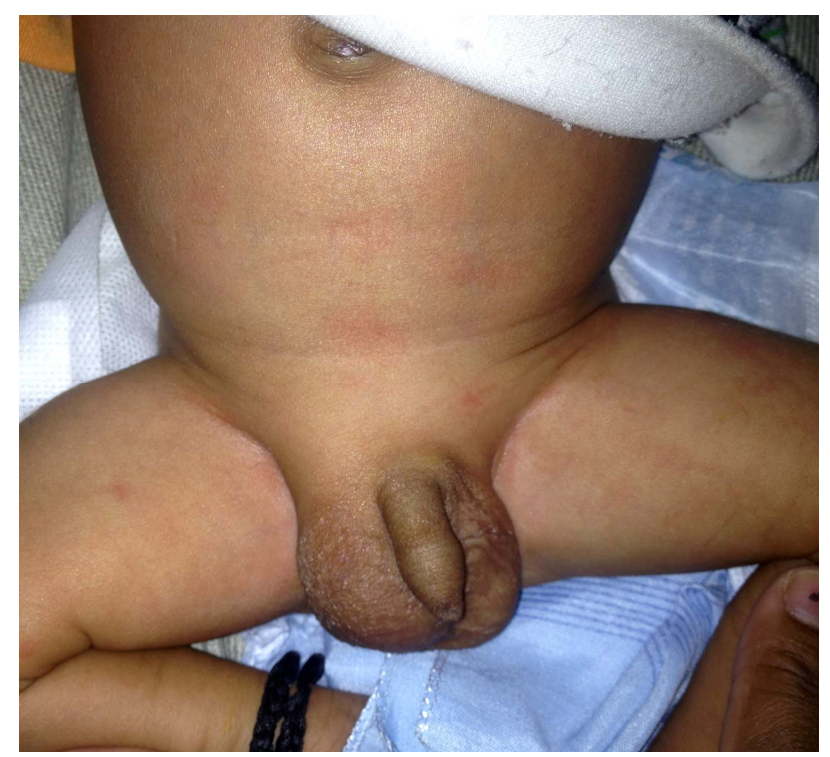

Figure 1. Irreducible swelling of right inguino-scrotal region. 


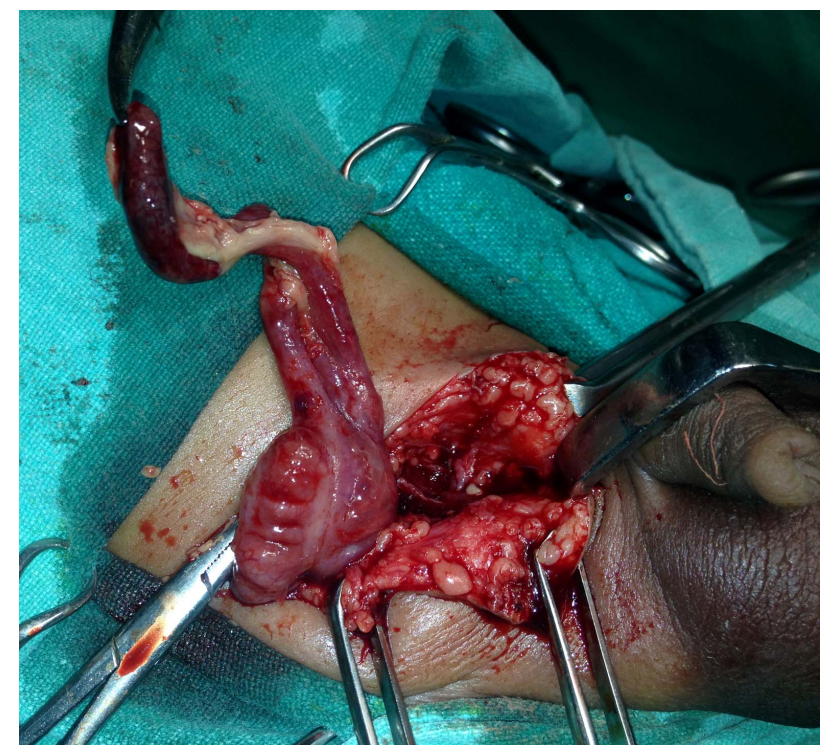

Figure 2. Gangrenous appendix with caecum.

dicitis which is rare in neonates, most cases of Amyand's hernia have been reported in premature neonates, infants and postmenopausal women. Majority of cases present as obstructed or strangulated inguinal hernia and even with perforation with generalized peritonitis, systemic signs and symptoms of appendicitis are rarely evident [3-5]. In our case the neonate presented with features of irreducible hernia and in spite of underlying gangrene of appendix, the features of bowel strangulation were completely masked. There was no distension of abdomen and child was accepting feeds normally. This caused an avoidable delay in surgical intervention. This was probably due to absence of luminal obstruction and sealing of toxic fluid in the sac due to dense adhesions. Presence of gangrenous appendix in Amyand's hernia is very rare [6]. Computer Tomography with Multi Planar Reconstruction is the most useful technique, to better visualize the appendix and its relationship with surrounding structures, thus aiding in confidently making the correct diagnosis pre-operatively [7]. Management depends on the state of the appendix, majority of authors agree that a normal appendix within the hernial sac does not require appendectomy [8,9]. In neonates with underlying appendicitis, herniotomy and appendicectomy should be performed $[9,10]$. In adults the repair of the hernia should be performed with Bassini or Shouldice techniques, without making use of synthetic meshes or plugs within the defect due to the high risk of suppuration of such materials [10].

\section{CONCLUSION}

Amyand's hernia is a rare entity with very difficult pre- operative diagnosis that does not effects management in majority of cases. But a great masquerader it can be in masking all the features of underlying bowel gangrene and delay in diagnosis can be detrimental in context of outcome. A high index of suspicion of Amyand's hernia with gangrene should be kept even if neonate present with irreducible hernia without features of strangulation to prevent avoidable increase in morbidity and mortality.

\section{REFERENCES}

[1] Vermillion, J.M., Abernathy, S.W. and Snyder, S.K. (1999) Laparoscopic reduction of Amyand's hernia. Hernia, 3, 159-160. http://dx.doi.org/10.1007/BF01195318

[2] Logan, M.T. and Nottingham, J.M. (2001) Amyand's hernia: A case report of an incarcerated and perforated appendix within an inguinal hernia and review of the literature. The American Journal of Surgery, 67, 628-629.

[3] D’Alia, C., Lo Schiavo, M.G., Tonante, A., et al. (2003) Amyand's hernia: Case report and review of the literature. Hernia, 7, 89-91. http://dx.doi.org/10.1007/s10029-002-0098-5

[4] Kueper, M.A., Kirschniak, A., Ladurner, R., Granderath, F.A. and Konigsrainer, A. (2007) Incarcerated recurrent inguinal hernia with covered and perforated appendicitis and periappendicular abscess: Case report and review of the literature. Hernia, 11, 189-191.

http://dx.doi.org/10.1007/s10029-006-0163-6

[5] Priego, P., Lobo, E., Moreno, I., Sanchez-Picot, S., Olarte, M.A.G., Alonso, N., et al. (2005) Acute appendicitis in an incarcerated crural hernia: Analysis of our experience. Revista Espanola de Enfermedades Digestivas, 97, 707715. http://dx.doi.org/10.4321/S1130-01082005001000004

[6] Breitenstein, S., Eisenbach, C., Wille, G. and Decurtins, M. (2005) Incarcerated vermiform appendix in a leftsided inguinal hernia. Hernia, 9, 100-102. http://dx.doi.org/10.1007/s10029-004-0263-0

[7] Laermans, S., Aerts, R. and De Man, R. (2007) Amyand's hernia: Inguinal hernia with acute appendicitis. JBR-BTR, 90, 524-525.

[8] Tycast, J.F., Kumpf, A.L., Schwartz, T.L. and Coln, C.E. (2008) Amyand's hernia: A case report describing laparoscopic repair in a pediatric patient. Journal of Pediatric Surgery, 43, 2112-2114. http://dx.doi.org/10.1016/j.jpedsurg.2008.07.032

[9] Meinke, A.K. (2007) Review article: Appendicitis in groin hernias. Journal of Gastrointestinal Surgery, 11, 13681372. http://dx.doi.org/10.1007/s11605-007-0160-9

[10] Ballas, K., Kontoulis, T., Skouras, C., et al. (2009) Unusual findings in inguinal hernia surgery: Report of 6 rare cases. Hippokratia, 13, 169-171. 